\title{
Mixing-Scale Dependent Dispersion For Transport in Heterogeneous Flows
}

\author{
M. Dentz ${ }^{1} \dagger$, F. P. J. de Barros ${ }^{2}$ \\ ${ }^{1}$ Spanish National Research Council, IDAEA-CSIC, c/ Jordi Girona 18-26, 08034 Barcelona, \\ Spain \\ ${ }^{2}$ Sonny Astani Dept. of Civil and Environmental Engineering, University of Southern \\ California, 3620 S. Vermont Avenue, KAP 224B, Los Angeles, CA 90089, USA
}

(23 August 2015)

\begin{abstract}
Dispersion quantifies the impact of subscale velocity fluctuations on the effective movement of particles and the evolution of scalar distributions in heterogeneous flows. Which fluctuation scales are represented by and what is the meaning of dispersion, depends on the definition of the subscale, or the corresponding coarse graining scale. We study here the dispersion effect due to velocity fluctuations that are sampled on the homogenization scale of the scalar distribution. This homogenization scale is identified with the mixing scale, the characteristic length below which the scalar is well mixed. It evolves in time as a result of local scale dispersion and the deformation of material fluid elements in the heterogeneous flow. The fluctuations scales below the mixing scale are equally accessible to all scalar particles, and thus contribute to enhanced scalar dispersion and mixing. We focus here on transport in steady spatially heterogeneous flow fields such as porous media flows. The dispersion effect is measured by mixing-scale dependent dispersion coefficients, which are defined through a filtering operation based on the evolving mixing scale. This renders the coarse grained velocity as a function of time, which evolves as velocity fluctuation scales are assimilated by the expanding scalar. We study the behavior of the mixing-scale dependent dispersion coefficients for transport in a random shear flow and in heterogeneous porous media. Using a stochastic modeling framework, we derive explicit expressions for their time behavior. The dispersion coefficients evolve as the mixing scale scans through the pertinent velocity fluctuation scales, which reflects the fundamental role of the interaction of scalar and velocity fluctuation scales on solute mixing and dispersion.
\end{abstract}

Key words: Mixing and Dispersion, Porous Media, Mixing Scale, Stochastic Modeling, Coarse Graining

\section{Introduction}

Flow heterogeneity impacts the spreading and mixing of particles and scalars. Velocity fluctuations lead to increased spread and dispersion of a transported scalar (Taylor 1953; Brenner 1980). The associated deformation of material fluid elements steepens concentration gradients, which together with local scale dispersive mass transfer generates increased scalar mixing (Villermaux \& Duplat 2003; Le Borgne et al. 2013). These processes control the large scale dynamics of scalar transport and are key for the understanding of contaminant levels, chemical reaction kinetics and bio-chemical activity in

$\dagger$ Email address for correspondence: marco.dentz@csic.es 
natural and engineered media (Tél et al. 2005; Dentz et al. 2011). The impact of velocity fluctuations on effective particle motion, and the global evolution of a transported scalar has been quantified in terms of eddy dispersion and macrodispersion coefficients (Dagan 1987; Weiss \& Provenzale 2008; Rubin et al. 1999). The rationale in such approaches is to coarse grain the particle motion by separating the Lagrangian velocity into a deterministic part and stochastic fluctuations, which give rise to a macroscopic dispersion effect.

We focus here on transport in steady, that means time-independent, spatially heterogeneous velocity fields as realized for Darcy scale flow and transport through heterogeneous porous media (Bear 1972). Spatial variability in hydraulic conducitvity here gives rise to fluctuations in the flow velocity, which is described by the Darcy equation (Bear 1972). As outlined above, the quantification of the impact of velocity fluctuations on large scale transport (this means larger than the characteristic heterogeneity scale) in terms of macrodispersion coefficients has been the subject of intense research over the last four decades (Gelhar \& Axness 1983; Dagan 1987; Rubin et al. 1999). Alternatively, a coarse graining scale may be defined by a spatial resolution scale which is determined by sampling and characterization strategies of spatial medium and flow heterogeneity, or by computational constraints, which require coarse numerical grid blocks (Beckie 1996; Beckie et al. 1996; Mehrabi \& Sahimi 1997; Rubin et al. 1999; de Barros \& Rubin 2011). As a consequence, large scale velocity variability is deterministically captured on the constant coarse graining, or blockscale, see Figure 1, whereas the subscale (below the coarse graining scale) variability remains unresolved and is modeled as a (correlated) stochastic fluctuation. The impact of the coarse graining scale on the resulting macroscale dispersion coefficient has been studied both in the framework of stochastic (Rubin et al. 1999) as well as volume averaging (Wood et al. 2003).

A key question here is, whether the erratic subscale velocity fluctuations contribute to increased particle and scalar dispersion and mixing, or rather contribute to advective spreading. This depends on the equal availability of the pertinent velocity fluctuations to the solute particles. Recall that dispersive particle motion can be modeled by the Langevin equation (Risken 1996)

$$
\frac{d \mathbf{x}(t)}{d t}=\overline{\mathbf{v}}+\mathbf{v}^{\prime}(t)
$$

with $\overline{\mathbf{v}}$ an average particle velocity and $\mathbf{v}^{\prime}(t)$ the random velocity fluctuation. This approach assumes that the particles have access to the full velocity fluctuation spectrum and thus are statistically equal. The dispersion coefficients are given by the Kubo formula as (Kubo et al. 1991)

$$
D_{i i}(t)=\int_{0}^{t} d t^{\prime}\left\langle v_{i}(t) v_{i}\left(t^{\prime}\right)\right\rangle,
$$

where the angular brackets denote the average over all realizations of $\mathbf{v}(t)$. For the particles to have equal access to the velocity fluctuations below the coarse-grained scale, it is required that they are able to sample the pertinent velocity fluctuation scales. This is equivalent to the requirement that the coarse-grained scale is well mixed. The mixing time for a block scale $\lambda_{b}$ due to local scale dispersive mass transfer may be estimated as $\tau_{b}=\lambda_{b}^{2} / D$ with $D$ the dispersion coefficient. In heterogenous flows, there is a competition between dispersive expansion and advective deformation of material fluid elements with the opposite effect (Villermaux \& Duplat 2006; Le Borgne et al. 2011). Thus, it can be expected that the mixing time is actually larger than the dispersion time $\tau_{b}$ over a block 
scale. For times smaller than the mixing time $t<\tau_{b}$, the dispersion coefficients measure rather advective solute spreading, or the advective deformation of the solute distribution, than mixing and dispersion. The impact of advective heterogeneity scales above the wellmixed scale, however, should be represented explicitly to adequately capture the impact of fluid deformation on solute mixing (Villermaux 2012; Le Borgne et al. 2013).

Instead of a constant blockscale to coarse grain the particle motion, we focus now on the physical length scales below which the scalar is well mixed as natural coarse graining scales. As indicated above, the evolution of such a mixing scale is governed by two processes, the advective deformation of material fluid elements, which steepens concentration gradients due to compression, and local scale dispersion, which seeks to increase the volume occupied by the scalar. In order to illustrate these mechanisms we consider first the expansion of a scalar due to dispersion only. The evolution of the characteristic scale is given by $d \lambda / d t=D / \lambda$. If on the other hand, we consider the evolution of the width of a material element in the absence of diffusion, we obtain from volume conservation $d \lambda / d t=-\gamma \lambda$, where $\gamma$ is the stretching rate of the material element. This relation is a direct consequence of volume preservation in the divergence-free flow. Combining the two mechanisms, one may write heuristically (Villermaux \& Duplat 2006; Villermaux 2012)

$$
\frac{1}{\lambda(t)} \frac{d \lambda(t)}{d t} \sim-\gamma(t)+\frac{D}{\lambda(t)^{2}}
$$

The stretching rate $\gamma(t)$ is in general a function of time. For chaotic flows, which are characterized by exponential stretching, $\gamma$ is constant. In this case, compression and dispersive expansion equilibrate at the Batchelor scale $\lambda_{B}=\sqrt{D / \gamma}$ (Batchelor 1959; Villermaux \& Duplat 2006). The steady flow fields under consideration in this paper are typically shear dominated and characterized by algebraic stretching, which implies that the stretching rate $\gamma(t) \sim 1 / t$ (Villermaux 2012; Le Borgne et al. 2013, 2015).

The different length scales, the constant blockscale $\lambda_{b}$, the dynamic mixing scale $\lambda(t)$ as well as the plume spreading scale are illustrated schematically in Figure 1, which shows a heterogeneous mixture evolving in a spatially fluctuating flow field. The mixture is clearly not well mixed on the spreading scale and characterized by lacunarities and spatial concentration heterogeneity. For the chosen constant block scale, we observe similar phenomena. At the time of the snapshot, the blockscale is larger than the mixing scale, and not all velocity fluctuations below $\lambda_{b}$ contribute to solute mixing and dispersion. The mixing scale, on the other hand marks the scale below which the mixture can be assumed uniform. Thus, it delineates the velocity fluctuation scales that contribute to solute dispersion, and separates them from the fluctuation scales that spread and deform the mixture into a laminar structure.

In this paper, we use the mixing scale to identify and quantify the fluctuation scales that can be represented by and contribute to dispersive particle motion. We contrast the distribution of velocity fluctuation scales with the evolution of the mixing scale, and introduce a filtering operation to separate random subscale fluctuations from deterministic large scale variability. A stochastic model seems to be a natural framework for the systematic quantification of mixing-scale dependent dispersion. We derive explicit expressions for the average dispersion coefficients, and discuss their evolution in the light of the assimilation of heterogeneity scales due to the evolving mixing scale.

The following section provides some background on the flow and transport model under consideration as well as the stochastic modeling approach to systematically capture the impact of flow heterogeneity on particle and scalar dispersion. Section 3 discusses the distribution of velocity fluctuation scales in the light of the velocity spectrum, and 


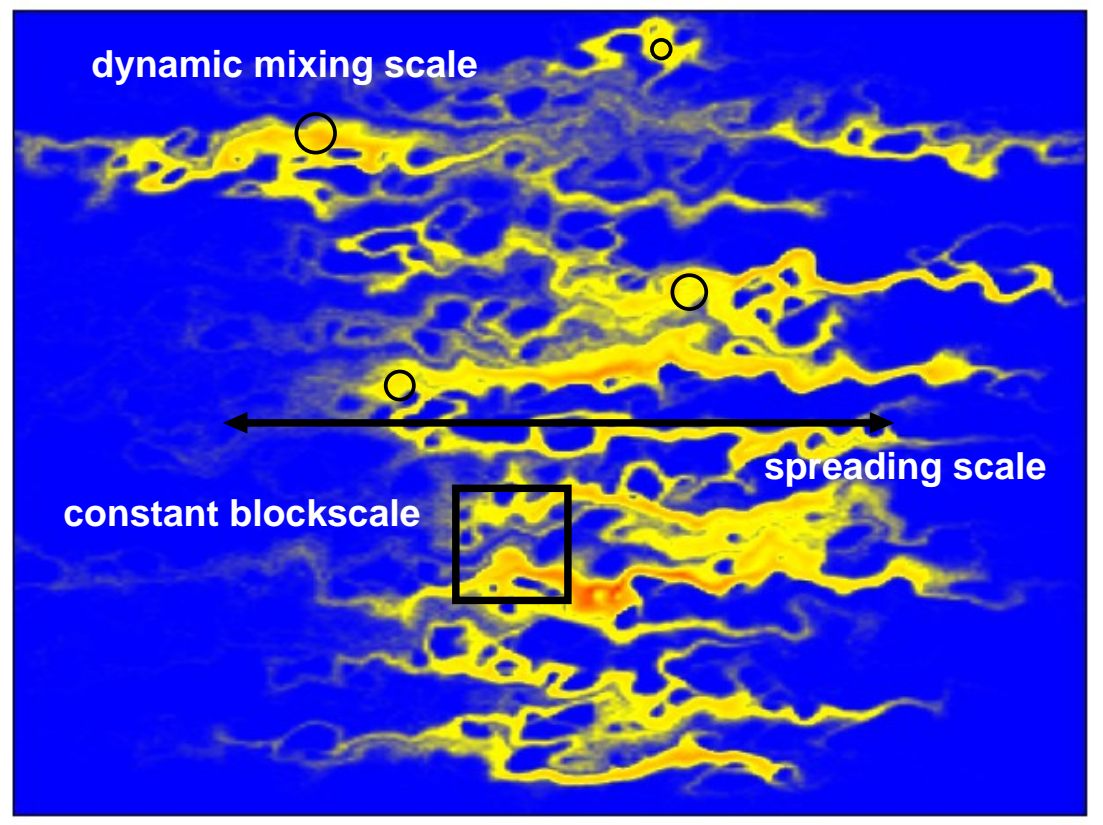

FIGURE 1. Illustration of the dispersion of a scalar transported in a two-dimensional spatially variable steady flow field. The scalar field is characterized by lacunarities and concentration heterogeneity on the spreading scale. The scalar distribution is uniform below the dynamic mixing scale, but not necessarily on the constant blockscale.

presents the evolution equation for the mixing scale as a result of advective deformation and dispersive expansion. Section 4 describes the coarse graining of the particle motion using the evolving mixing scale and derives the filtered dispersion coefficients, which account for the impact of sub-mixing scale velocity fluctuations on scalar and particle dispersion. Section 5 applies the developed concept to the cases of dispersion in a random shear flow, and heterogeneous porous media flows.

\section{Flow and Transport Model}

The spatio-temporal evolution of a scalar $c(\mathbf{x}, t)$ in the divergence-free flow field $\mathbf{v}(\mathbf{x})$ through a Darcy-scale heterogeneous porous medium is governed by (Bear 1972)

$$
\frac{\partial c(\mathbf{x}, t)}{\partial t}+\mathbf{v}(\mathbf{x}) \cdot \nabla c(\mathbf{x}, t)-\nabla \cdot \mathbf{D} \nabla c(\mathbf{x}, t)=0 .
$$

Porosity is assumed to be constant. It is set here equal to 1 , which is equivalent to rescaling time. The flow velocity $\mathbf{v}(\mathbf{x})$ is given by the Darcy equation, and the local scale dispersion tensor $\mathbf{D}$ quantifies the impact of molecular diffusion and pore-scale velocity fluctuations on solute dispersion on the Darcy scale (Bear 1972). For simplicity, we assume here that $\mathbf{D}$ is constant and isotropic, $D_{i j}=D \delta_{i j}$. The foundations of the Darcy scale transport model (2.1) can be found in the books by Bear (1972), Whitaker (1999) and Hornung (1997), for example. For a discussion on its validity and limitations, we refer the reader to the review by Dentz et al. (2011) and references therein. We consider the normalized initial condition $c(\mathbf{x}, t=0)=c_{0}(\mathbf{x})$. 
The scalar transport problem (2.1) can be formulated equivalently in terms of the Langevin equation

$$
\frac{d \mathbf{x}(t)}{d t}=\mathbf{v}[\mathbf{x}(t)]+\boldsymbol{\xi}(t) .
$$

The initial particle positions are $\mathbf{x}(t=0)=\mathbf{x}^{\prime}$ with $\mathbf{x}^{\prime}$ distributed according to $c_{0}\left(\mathbf{x}^{\prime}\right)$. The Gaussian white noise $\boldsymbol{\xi}(t)$ is characterized by zero mean and covariance

$$
\left\langle\xi_{i}(t) \xi_{j}(t)\right\rangle=2 D \delta_{i j} \delta\left(t-t^{\prime}\right),
$$

where the angular brackets denotes the noise mean over all realizations of the random noise $\boldsymbol{\xi}(t), \delta_{i j}$ is the Kronecker delta and $\delta(t)$ the Dirac delta-distribution.

The heterogeneous velocity field $\mathbf{v}(\mathbf{x})$ is modeled as a stationary and ergodic random field. The mean flow is aligned with the 1-direction of the coordinate system, that is $\overline{\mathbf{v}(\mathbf{x})}=\bar{v} \mathbf{e}_{1}$. The ensemble mean in the following is represented by an overbar. The flow fluctuations $\mathbf{v}^{\prime}(\mathbf{x})=\mathbf{v}(\mathbf{x})-\overline{\mathbf{v}}$ have the covariance $\mathcal{C}_{i j}\left(\mathbf{x}-\mathbf{x}^{\prime}\right)=\overline{v_{i}^{\prime}(\mathbf{x}) v_{j}^{\prime}\left(\mathbf{x}^{\prime}\right)}$. Its Fourier transform is given by

$$
\tilde{\mathcal{C}}_{i j}(\mathbf{k})=\bar{v}^{2} \psi_{i j}(\mathbf{k}) \tilde{\mathcal{E}}(\mathbf{k}),
$$

where $\mathbf{k}$ is the wave vector. The projectors $\psi_{i j}(\mathbf{k})$ are a consequence of $\nabla \cdot \mathbf{v}(\mathbf{x})=0$. They satisfy $\sum_{i} k_{i} \psi_{i j}(\mathbf{k})=\sum_{j} k_{j} \psi_{i j}(\mathbf{k})=0$. The correlation spectrum, or power spectrum of the velocity fluctuations is denoted by $\tilde{\mathcal{E}}(\mathbf{k})$. Fourier transformed quantities in the following are marked by a tilde. We employ the definitions

$$
\tilde{f}(\mathbf{k})=\int d \mathbf{x} \exp (i \mathbf{k} \cdot \mathbf{x}) f(\mathbf{x}, t), \quad f(\mathbf{x})=\int_{k} \exp (-i \mathbf{k} \cdot \mathbf{x}) \tilde{f}(\mathbf{k}),
$$

for the Fourier transform and its inverse. Furthermore, we use the shorthand notation

$$
\int_{k}=\int \frac{d \mathbf{k}}{(2 \pi)^{d}}
$$

where $d$ denotes spatial dimensionality. Note that the covariance of the Fourier-transformed velocity fluctuations is given by

$$
\overline{v_{i}^{\prime}(\mathbf{k}) v_{j}^{\prime}\left(\mathbf{k}^{\prime}\right)}=(2 \pi)^{d} \delta\left(\mathbf{k}+\mathbf{k}^{\prime}\right) \tilde{\mathcal{C}}_{i j}(\mathbf{k}),
$$

where $d$ denotes the dimensionality of space. The Dirac-delta is a consequence of the translation invariance of the covariance function.

For flow in heterogeneous porous media, the divergence-free flow velocity $\mathbf{v}(\mathbf{x})$ is related to the medium heterogeneity through the Darcy equation $\mathbf{v}(\mathbf{x})=-\exp [f(\mathbf{x})] \nabla h(\mathbf{x})$ with $h(\mathbf{x})$ hydraulic head and $f(\mathbf{x})$ log-hydraulic conductivity. The latter is modeled here as a stationary and ergodic Gaussian random field. A perturbation analysis in the fluctuations of log-hydraulic conductivity about its constant ensemble mean, $f^{\prime}(\mathbf{x})=f(\mathbf{x})-\bar{f}$, relates the Fourier transformed velocity fluctuation $\tilde{v}_{i}^{\prime}(\mathbf{k})$ to $\tilde{f}^{\prime}(\mathbf{k})$ as (Gelhar \& Axness 1983; Dagan 1987)

$$
\tilde{v}_{i}^{\prime}(\mathbf{k})=\bar{v}\left(\delta_{1 i}-\frac{k_{1} k_{i}}{\mathbf{k}^{2}}\right) \tilde{f}^{\prime}(\mathbf{k})
$$

which implies that $\mathbf{v}(\mathbf{x})$ follows also Gaussian statistics. Correspondingly, the covariance of $\tilde{\mathbf{v}}^{\prime}(\mathbf{k})$ is given by

$$
\overline{v_{i}^{\prime}(\mathbf{k}) v_{j}^{\prime}\left(\mathbf{k}^{\prime}\right)}=\bar{v}^{2}\left(\delta_{1 i}-\frac{k_{1} k_{i}}{\mathbf{k}^{2}}\right)\left(\delta_{1 j}-\frac{k_{1} k_{j}}{\mathbf{k}^{2}}\right) \overline{\tilde{f}^{\prime}(\mathbf{k}) \tilde{f}^{\prime}\left(\mathbf{k}^{\prime}\right)}
$$




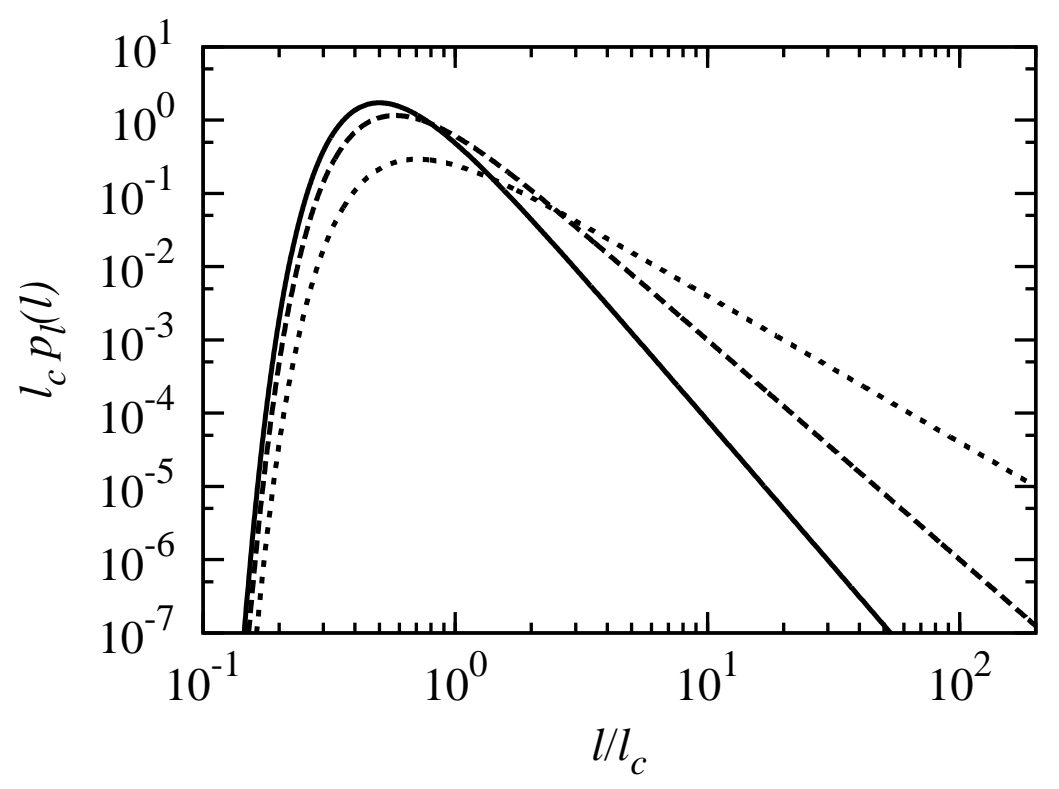

FiguRE 2. Distribution $p_{l}(l)$ of fluctuation length scales given by (3.3) for (solid line) $d=3$, (dashed line) $d=2$ and (dotted lines) $d=1$ spatial dimensions.

which renders the projectors as $\psi_{i j}(\mathbf{k})=\left(\delta_{1 i}-k_{1} k_{i} / \mathbf{k}^{2}\right)\left(\delta_{1 j}-k_{1} k_{j} / \mathbf{k}^{2}\right)$. Thus, the correlation spectrum $\tilde{\mathcal{E}}(\mathbf{k})$ is equal to the Fourier transform of the covariance function of the log-hydraulic conductivity. For illustration, in the following we employ the isotropic Gaussian spectrum (Rubin 2003)

$$
\tilde{\mathcal{E}}(\mathbf{k})=\sigma_{\mathcal{E}}^{2}(2 \pi)^{d / 2} l_{c}^{d} \exp \left(-\mathbf{k}^{2} l_{c}^{2} / 2\right)
$$

with $\sigma_{\mathcal{E}}^{2}$ the disorder variance and $l_{c}$ the correlation length scale.

The characteristic transport time scales are given here by the characteristic dispersion time over the correlation scale $l_{c}$, which is defined by $\tau_{D}=l_{c}^{2} / D$, and the advection time scale $\tau_{v}=l_{c} / \bar{v}$, which quantifies the characteristic advection time over the correlation distance $l_{c}$. These time scales are compared by the Péclet number $P e=\tau_{D} / \tau_{v}$, which quantifies the relative strength of dispersive and advective transport.

\section{Fluctuation and Mixing Scales}

In this section, we discuss the characteristic velocity fluctuation scales in terms of the velocity spectrum, and describe the evolution of the mixing scale as a result of the competition between dispersive expansion and advective compression.

\subsection{Fluctuation Scales}

The distribution of heterogeneity scales $l$ is encoded in the correlation spectrum $\tilde{\mathcal{E}}(\mathbf{k})$, which quantifies the amplitude associated with the wavenumber $\mathbf{k}$. Notice that $|\mathbf{k}|$ is related to the fluctuation length scales $l$ by $l=1 /|\mathbf{k}|$. Thus, we may obtain the PDF $p_{l}(l)$ of fluctuation length scale from the PDF of wave numbers $p_{k}(\mathbf{k})$ through the mapping $l=1 /|\mathbf{k}|$. The PDF of wave numbers is given in terms of the correlation spectrum $\tilde{\mathcal{E}}(\mathbf{k})$ 


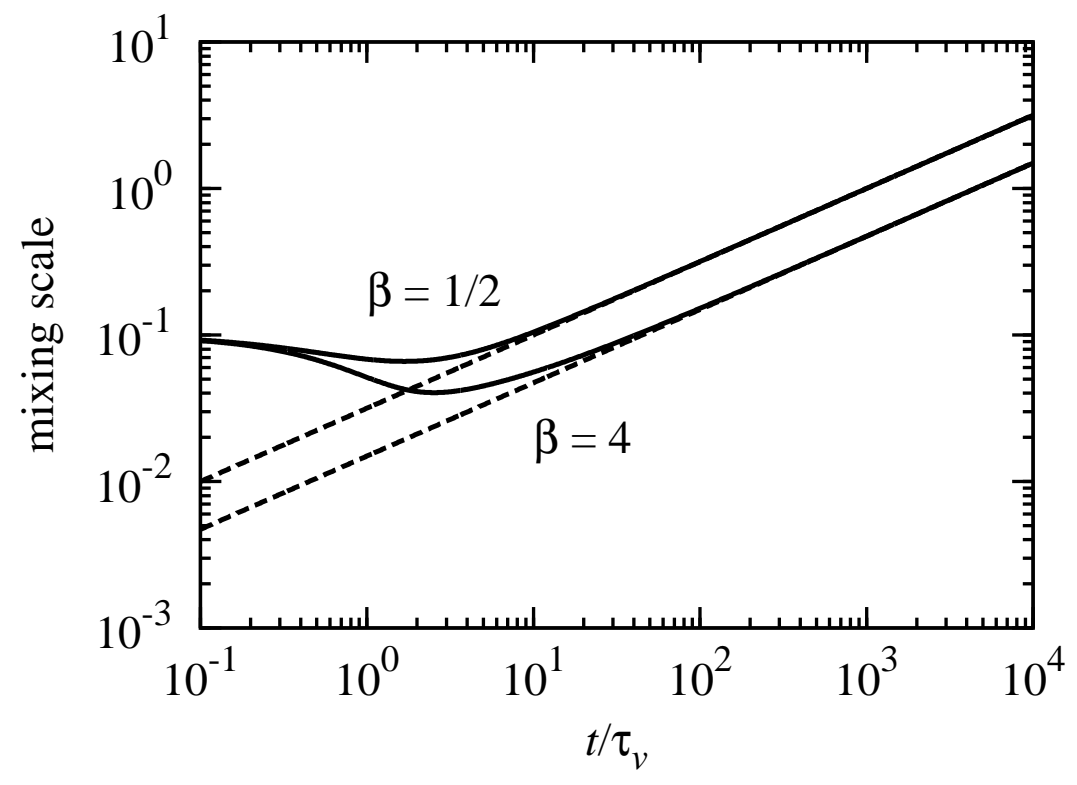

Figure 3. Evolution of the mixing scale $\lambda(t)$ for $P e=10^{3}$ and $\lambda(t=0) / l_{c}=10^{-1}$. The dashed lines show the dispersive $\sqrt{2 D t f_{\beta}}$ behavior.

as

$$
p_{k}(\mathbf{k})=\tilde{\mathcal{E}}(\mathbf{k}) / \int d \mathbf{k} \tilde{\mathcal{E}}(\mathbf{k})
$$

Specifically, for the Gaussian spectrum $(2.10), p_{k}(k)$ is given by

$$
p_{k}(k)=\frac{l_{c}^{d} \exp \left(-\mathbf{k}^{2} l_{c}^{2} / 2\right)}{(2 \pi)^{d / 2}}
$$

Thus, we obtain from the mapping $l=1 /|\mathbf{k}|$ the explicit PDF of fluctuation scales

$$
p_{l}(l)=\frac{l_{c}^{d}}{l^{d+1}} \frac{\Omega_{d} \exp \left[-l_{c}^{2} /\left(2 l^{2}\right)\right]}{(2 \pi)^{d / 2}},
$$

where $\Omega_{d}$ is the surface area of the $d$-dimensional unit sphere. The scale distribution of different correlation spectra can be determined in the same way. The scale distribution (3.3) is illustrated in Figure 2. It is cut-off exponentially at scales $l \ll l_{c}$ and falls off algebraically for $l \gg l_{c}$. It has a maximum at $l_{\max }=l_{c} / \sqrt{d+1}$. Note that a scalar can be assumed to have sampled a representative part of the spatial heterogeneity when it has swept about 10 heterogeneity length scales. Thus, we define now as a minimum heterogeneity scale $l_{m}$ the length $l \leqslant l_{\max }$ at which $p_{l}\left(l_{m}\right)=p_{l}\left(10 l_{c}\right)$. For $d=3$, we obtain $l_{m}=0.18 l_{c}$, for $d=2, l_{m}=0.2 l_{c}$ and for $d=1, l_{m}=0.26 l_{c}$. In the same way as heterogeneity scales $l>10 l_{c}$ do not play a role for scalar transport, scales below $l_{m}$ have only limited impact.

\subsection{Mixing Scale}

The fluctuation scales $l$ of the heterogeneous flow field are contrasted with the mixing scale $\lambda(t)$, which can be defined as the characteristic gradient scale of a heterogeneous 
mixture (Villermaux \& Duplat 2006; Le Borgne et al. 2011). Thus, it marks the scale below which the scalar mixture can be assumed homogeneous. The temporal evolution of the mixing scale is governed by the competition of dispersion, which tends to increase the mixing scale, and compression due to deformation of material segments, which tends to decrease it. This can be illustrated by considering solute transport in the coordinate system attached to the material fluid element that deforms as it is transported in the flow field. The concentration $\hat{c}(z, t)$ across a material fluid element evolves according to the advection dispersion equation (Ranz 1979; Meunier \& Villermaux 2010)

$$
\frac{\partial \hat{c}(z, t)}{\partial t}-\gamma(t) z \frac{\partial \hat{c}(z, t)}{\partial z}-D \frac{\partial^{2} \hat{c}(z, t)}{\partial z^{2}}=0
$$

The spatial variance

$$
\kappa(t)=\int d z z^{2} \hat{c}(z, t) / \int d z \hat{c}(z, t) .
$$

is a measure for the square width of the solute distribution across the deformed material element. It satisfies the evolution equation (Tennekes \& Lumley 1972; de Barros et al. 2012)

$$
\frac{d \kappa(t)}{d t}=-2 \gamma(t) \kappa(t)+2 D
$$

We set now the mixing scale $\lambda(t)$ equal to the width of the scalar concentration in a deformed material strip, which captures the competition between dispersive expansion and advective compression, $\lambda(t)=\sqrt{\kappa(t)}$. It satisfies the equation (Villermaux \& Duplat 2006; Villermaux 2012)

$$
\frac{1}{\lambda(t)} \frac{d \lambda(t)}{d t}=-\gamma(t)+\frac{D}{\lambda(t)^{2}}
$$

which in the Introduction was phenomenologically motivated. For the steady flow fields under consideration here, stretching and compression of a material element is algebraic (Villermaux 2012) such that $\gamma(t) \sim \beta t^{-1}$. For the choice of $\gamma(t)=\beta /\left[\tau_{v}\left(\beta+t / \tau_{v}\right)\right]$, the evolution of the mixing scale is determined by solving Equation (3.7) subject to the initial condition $\lambda(t=0)=\lambda_{0}$. We obtain after a quick calculation

$$
\lambda(t)=\sqrt{2 D t f_{\beta}\left(1+\beta \tau_{v} / t\right)+\left(\frac{\beta}{t / \tau_{v}+\beta}\right)^{2 \beta}\left(\lambda_{0}^{2}-2 D \beta f_{\beta}^{-1} \tau_{v}\right)}
$$

with $f_{\beta}=1 /(1+2 \beta)$. Figure 3 illustrates the evolution of $\lambda(t)$ with time. At short times, the mixing scale decreases as a result of compression until it assumes a minimum at the mixing time $\tau_{m}=\tau_{v}\left(\lambda_{0}^{2} / D \tau_{v}\right)^{1 /(2 \beta+1)}$ (Villermaux 2012), at which advective compression and dispersive expansion equilibrate. For times larger than the mixing time, $t>\tau_{m}$, dispersive expansion prevails and $\lambda(t)$ evolves dispersively, that is, $\lambda(t) \sim \sqrt{2 D t f_{\beta}}$. Note that the dispersive long-time behavior is impacted on by compression through a reduction in the dispersion coefficient by the factor $0<f_{\beta} \leqslant 1$.

\section{Mixing-Scale Dependent Dispersion}

We separate the velocity fluctuations below and above the mixing scale $\lambda(t)$ in order to determine the dispersion effect of flow fluctuations below the homogenization scale of the transported scalar. The coarse-grained velocity field $\mathbf{v}_{>}(\mathbf{x}, t)$ is defined by spatial 
filtering as

$$
\mathbf{v}_{>}(\mathbf{x}, t)=\int d \mathbf{r} \mathcal{F}(\mathbf{x}-\mathbf{r}, t) \mathbf{v}(\mathbf{r})
$$

where $\mathcal{F}(\mathbf{x}, t)$ is a suitably chosen filter (Beckie et al. 1996; Rubin et al. 1999). This approach is similar to the large eddy simulation methodology used in geophysical fluid dynamics (Beckie et al. 1996; Ferziger \& Perić 2002), which, however, uses a constant filter. It is interesting to note that through the use of the time-dependent filtering scale $\lambda(t)$, the coarse-grained velocity field $\mathbf{v}_{>}(\mathbf{x}, t)$ is a function of time, even though the underlying $\mathbf{v}(\mathbf{x})$ is steady. The time-dependence reflects the fact that $\mathbf{v}_{>}(\mathbf{x}, t)$ acquires additional fluctuation modes as the mixing scale $\lambda(t)$ evolves. The coarse-graining operation reads in Fourier space as

$$
\tilde{\mathbf{v}}_{>}(\mathbf{k}, t)=\tilde{\mathcal{F}}(\mathbf{k}, t) \tilde{\mathbf{v}}(\mathbf{k}) .
$$

In the following, we employ the isotropic Gaussian filter

$$
\mathcal{F}(\mathbf{x}, t)=\frac{\exp \left[-\frac{\mathbf{x}^{2}}{2 \lambda(t)^{2}}\right]}{\left[2 \pi \lambda(t)^{2}\right]^{d / 2}}, \quad \tilde{\mathcal{F}}(\mathbf{k}, t)=\exp \left[-\frac{\mathbf{k}^{2} \lambda(t)^{2}}{2}\right]
$$

The subscale scale velocity fluctuations are defined by $\mathbf{v}_{<}(\mathbf{x}, t)=\mathbf{v}(\mathbf{x})-\mathbf{v}_{>}(\mathbf{x}, t)$. Notice that the ensemble mean of the coarse grained flow velocity $\mathbf{v}_{>}(\mathbf{x}, t)$ is equal to the mean flow velocity

$$
\overline{\mathbf{v}_{>}(\mathbf{x}, t)}=\int d \mathbf{r} \mathcal{F}(\mathbf{x}-\mathbf{r}, t) \overline{\mathbf{v}(\mathbf{r})}=\overline{\mathbf{v}}
$$

because the filter $\mathcal{F}(\mathbf{x}, t)$ is normalized. As an immediate consequence, we obtain that $\overline{\mathbf{v}_{<}(\mathbf{x}, t)}=\mathbf{0}$.

We write now the fine scale Langevin equation (2.2) tautologically in the form

$$
\frac{d \mathbf{x}(t)}{d t}=\mathbf{v}_{>}[\mathbf{x}(t), t]+\boldsymbol{\zeta}(t),
$$

where the effective noise $\boldsymbol{\zeta}(t)=\mathbf{v}_{<}[\mathbf{x}(t), t]+\boldsymbol{\xi}(t)$ represents the sub-scale heterogeneity. The noise mean of $\zeta_{i}(t)$ can be expanded as

$$
\left\langle\zeta_{i}(t)\right\rangle=\int_{k} \mathcal{A}(\mathbf{k}, t) \tilde{v}_{i}(\mathbf{k}) \tilde{c}\left(\mathbf{k}, t \mid \mathbf{x}_{0}\right)
$$

where $\tilde{c}\left(\mathbf{k}, t \mid \mathbf{x}_{0}\right)=\langle\exp [-i \mathbf{k} \cdot \mathbf{x}(t)]\rangle$ is equal to the Fourier transform of scalar concentration evolving from a point source at $\mathbf{x}(t=0)=\mathbf{x}_{0}$. We defined for convenience

$$
\mathcal{A}(\mathbf{k}, t)=1-\tilde{\mathcal{F}}(\mathbf{k}, t) .
$$

Analogously, we obtain for the noise cross-moments

$$
\begin{aligned}
& \left\langle\zeta_{i}(t) \zeta_{j}\left(t^{\prime}\right)\right\rangle=2 D_{i i} \delta_{i j} \delta\left(t-t^{\prime}\right)+\int d \mathbf{x}^{\prime} \int_{k} \int_{k^{\prime}} \int_{k^{\prime \prime}} \mathcal{A}(\mathbf{k}, t) \mathcal{A}\left(\mathbf{k}^{\prime}, t^{\prime}\right) \\
& \times \tilde{v}_{i}(\mathbf{k}) \tilde{v}_{j}\left(\mathbf{k}^{\prime}\right) \tilde{c}\left(-\mathbf{k}, t-t^{\prime} \mid \mathbf{x}^{\prime}\right) \exp \left(i \mathbf{k}^{\prime \prime} \cdot \mathbf{x}^{\prime}\right) \tilde{c}\left(-\mathbf{k}^{\prime}-\mathbf{k}^{\prime \prime}, t^{\prime} \mid \mathbf{x}_{0}\right) .
\end{aligned}
$$

The coarse-scale particle trajectory $\mathbf{x}_{>}(t)$ in a single disorder realization is defined by

$$
\mathbf{x}_{>}(t)=\int_{0}^{t} d t^{\prime} \mathbf{v}_{>}\left[\mathbf{x}\left(t^{\prime}\right), t^{\prime}\right]
$$


Per (4.6), the noise mean of the mesoscopic noise term $\boldsymbol{\zeta}(t)$ is in general non-zero, and therefore, the noise mean trajectory and the coarse-scale noise mean trajectories do not coincide. Their difference $\delta\langle\mathbf{x}(t)\rangle \equiv\langle\mathbf{x}(t)\rangle-\left\langle\mathbf{x}_{>}(t)\right\rangle$ is given by

$$
\delta\langle\mathbf{x}(t)\rangle=\int_{0}^{t} d t^{\prime}\left\langle\boldsymbol{\zeta}\left(t^{\prime}\right)\right\rangle
$$

The effect of fine-scale fluctuations on particle dispersion can be quantified in terms of the mesoscale noise correlation by using the Kubo formula as (Kubo et al. 1991)

$$
\mathcal{D}_{i i}(t)=\int_{0}^{t} d t^{\prime}\left[\left\langle\zeta_{i}(t) \zeta_{i}\left(t^{\prime}\right)\right\rangle-\left\langle\zeta_{i}(t)\right\rangle\left\langle\zeta_{i}\left(t^{\prime}\right)\right\rangle\right]_{0},
$$

where $\mathcal{D}_{i i}(t)$ are the single realization mixing-scale dependent dispersion coefficients. The square brackets with subindex 0 denote the averaging over the normalized distribution $c_{0}(\mathbf{x})$ of initial particle positions $\mathbf{x}(t=0)=\mathbf{x}_{0}$,

$$
\left[\tilde{c}\left(\mathbf{k}, t \mid \mathbf{x}^{\prime}\right)\right]_{0}=\int d \mathbf{x}^{\prime} c_{0}\left(\mathbf{x}^{\prime}\right) \tilde{c}\left(\mathbf{k}, t \mid \mathbf{x}^{\prime}\right) .
$$

The stochastic approach defines dispersion coefficients as suitably chosen ensemble averages. The effective dispersion coefficient is given by the ensemble average of (4.11) as

$$
D_{i i}^{\mathrm{eff}}(t) \equiv \overline{\mathcal{D}_{i i}(t)} \text {. }
$$

Alternatively, we define the ensemble dispersion coefficient, which measures the dispersion effects of velocity fluctuations relative to the ensemble mean noise $\overline{\langle\boldsymbol{\zeta}(t)\rangle}$. Due to the translation invariance of $\mathbf{v}(\mathbf{x})$, the ensemble average over the mesoscale noise $\boldsymbol{\zeta}(t)$ is zero, $\overline{\langle\boldsymbol{\zeta}(t)\rangle}=\mathbf{0}$. Thus, the ensemble dispersion coefficient is given by

$$
D_{i i}^{\mathrm{ens}}(t)=\int_{0}^{t} d t^{\prime} \overline{\left[\left\langle\zeta_{i}(t) \zeta_{j}\left(t^{\prime}\right)\right\rangle\right]_{0}}
$$

Notice that the difference between the ensemble and effective dispersion coefficients quantifies the rate of change of the variance of the trajectory fluctuations (4.10)

$$
\frac{1}{2} \frac{d \overline{\left[\delta\left\langle x_{i}(t)\right\rangle^{2}\right]_{0}}}{d t}=\int_{0}^{t} d t^{\prime} \overline{\left[\left\langle\zeta_{i}(t)\right\rangle\left\langle\zeta_{j}\left(t^{\prime}\right)\right\rangle\right]_{0}}=D_{i i}^{\mathrm{ens}}(t)-D_{i i}^{\mathrm{eff}}(t) .
$$

Thus, it is also a measure for the uncertainty in the coarse-grained particle positions. The difference between the unfiltered effective and ensemble averaged dispersion coefficients has been discussed in the past (Kitanidis 1988; Dagan 1991; Fiori 1998; Dentz et al. 2000).

\section{Dispersion Behavior}

In this section we illustrate the behavior of the mixing-scale dependent dispersion coefficients for transport in a random shear flow and in the flow through a heterogeneous porous medium. 


\subsection{Random Shear Flow}

We first consider the problem of dispersion in a random shear flow as it occurs in a randomly stratified porous medium (Matheron \& de Marsily 1980). It is characterized by the flow velocity $\mathbf{v}(\mathbf{x})=\mathbf{e}_{1} v\left(x_{2}\right)$ with $\mathbf{e}_{1}$ the unit vector in 1 -direction. We assume that the hydraulic gradient is aligned with the direction of stratification and points in the 1 -direction of the coordinate system. For this case, the projectors $\psi_{i j}(\mathbf{k})=\delta_{1 i} \delta_{1 j}$, and thus, the velocity covariance function (2.4) reduces to

$$
\tilde{\mathcal{C}}\left(k_{2}\right)=\bar{v}^{2} \tilde{\mathcal{E}}\left(k_{2}\right) \text {. }
$$

As outlined in Section 3, for the Gaussian spectrum (2.10), the fluctuation spectrum is given by (3.3) for $d=1$. Transport in random shear flows such as the one under consideration here (Matheron \& de Marsily 1980), has been studied in the literature as an exactly solvable model for heterogeneous aquifers (Zavala-Sanchez et al. 2009, and literature therein) characterized by stratification in the horizontal plane. Both steady and unsteady random shear flows have been studied in the physics literature as simplified models for heterogenous and turbulent flows (Bouchaud \& Georges 1990).

The stretching of a material fluid element in the random shear flow is a non-sequential process (Duplat et al. 2010) in the sense that the shear properties are constant in a given stratum, but vary between strata. The elongation of a material fluid element due to the random shear $\sigma=\partial v\left(x_{2}\right) / \partial x_{2}$ relative to its initial length is given by

$$
\rho(t)=\sqrt{1+\sigma^{2} t^{2}}
$$

Note that the shear rate depends on the vertical position, and is a random space function as the stratified flow velocity $v\left(x_{2}\right)$. The stretching rate $\gamma(t)=d \ln \rho(t) / d t$ now is given by

$$
\gamma(t)=\frac{\sigma^{2} t}{1+\sigma^{2} t^{2}}
$$

At times $t \gg \sigma^{-1}$ it is $\gamma(t) \approx 1 / t$. Inserting (5.3) into the evolution equation (3.7), we obtain the mixing scale

$$
\lambda(t)=\sqrt{\frac{2 D\left(t+\sigma^{2} t^{3} / 3\right)+\lambda_{0}^{2}}{1+\sigma^{2} t^{2}}} .
$$

For times $t>\left(\lambda_{0}^{2} / D \sigma^{2}\right)^{1 / 3}$, it behaves as $\lambda(t) \approx \sqrt{2 D t / 3}$. It is independent of the random shear rate $\sigma$.

In order to derive explicite expressions for the filtered dispersion coefficients, we substitute $\tilde{v}_{i}(\mathbf{k})=\delta_{i 1} \tilde{v}\left(k_{2}\right)$ into (4.6) and (4.8). This gives for the noise mean $\left\langle\zeta_{i}(t)\right\rangle$ and the cross moment $\left\langle\zeta_{i}(t) \zeta_{j}\left(t^{\prime}\right)\right\rangle$

$$
\begin{aligned}
\left\langle\zeta_{i}(t)\right\rangle & =\delta_{i 1} \int_{k_{2}} \mathcal{A}\left(k_{2}, t\right) \tilde{v}\left(k_{2}\right) \tilde{c}\left(k_{2}, t \mid a\right) \\
\left\langle\zeta_{i}(t) \zeta_{j}\left(t^{\prime}\right)\right\rangle & =2 D_{i i} \delta_{i j} \delta\left(t-t^{\prime}\right)+\delta_{i 1} \delta_{j 1} \int d a^{\prime} \int_{k_{2}} \int_{k_{2}^{\prime}} \int_{k_{2}^{\prime \prime}} \mathcal{A}\left(k_{2}, t\right) \mathcal{A}\left(k_{2}^{\prime}, t^{\prime}\right) \\
& \times \tilde{v}\left(k_{2}\right) \tilde{v}\left(k_{2}^{\prime}\right) \tilde{c}\left(-k_{2}, t-t^{\prime} \mid a^{\prime}\right) \exp \left(i k_{2}^{\prime \prime} a^{\prime}\right) \tilde{c}\left(-k_{2}^{\prime}-k_{2}^{\prime \prime}, t^{\prime} \mid a\right) .
\end{aligned}
$$

where $\tilde{c}\left(k_{2}, t \mid a\right)=\left\langle\exp \left[i k_{2} x_{2}(t)\right]\right\rangle$ is the Fourier transform of the scalar distribution for the random walk

$$
\frac{d x_{2}(t)}{d t}=\xi_{2}(t)
$$


with the initial position $x_{2}(t=0)=a$. It is given by

$$
\tilde{c}\left(k_{2}, t \mid a\right)=\exp \left(-D k_{2}^{2} t+i k_{2} a\right) .
$$

Using (5.6) together with (5.8) and (5.1) in (4.14) gives for the longitudinal ensemble dispersion coefficient

$$
D_{11}^{\mathrm{ens}}(t)=D+\bar{v}^{2} \int_{0}^{t} d t^{\prime} \int_{k_{2}} \mathcal{A}\left(k_{2}, t\right) \mathcal{A}\left(k_{2}, t^{\prime}\right) \tilde{\mathcal{E}}\left(k_{2}\right) \exp \left[-D k_{2}^{2}\left(t-t^{\prime}\right)\right] .
$$

The trajectory fluctuations (4.15) are obtained by using (5.5) together with (5.8) and (5.1)

$$
\frac{1}{2} \frac{d \overline{\delta\left\langle x_{i}(t)\right\rangle^{2}}}{d t}=\bar{v}^{2} \int_{0}^{t} d t^{\prime} \int_{k_{2}} \mathcal{A}\left(k_{2}, t\right) \mathcal{A}\left(k_{2}, t^{\prime}\right) \tilde{\mathcal{E}}\left(k_{2}\right) \exp \left[-D k_{2}^{2}\left(t+t^{\prime}\right)\right] .
$$

Using furthermore the definition (4.7) of $\mathcal{A}\left(k_{2}, t\right)$ and the Gaussian filter (4.3) with the mixing scale $\lambda(t)=\sqrt{2 D t / 3}$, the integrals in (5.9) and (5.10) can be evaluated explicitly. Thus, we obtain for $D_{11}^{\text {ens }}(t)$ the explicit expression

$$
\begin{aligned}
& D_{11}^{\mathrm{ens}}(t)=D+ \\
& \quad \sigma_{\mathcal{E}}^{2} \frac{\bar{v}^{2} l_{c}^{2}}{2 D}\left(\sqrt{1+\frac{8 t}{3 \tau_{D}}}+5 \sqrt{1+\frac{2 t}{3 \tau_{D}}}-3 \sqrt{1+\frac{4 t}{3 \tau_{D}}}-\sqrt{1+\frac{2 t}{\tau_{D}}}-2\right) .
\end{aligned}
$$

For the trajectory fluctuations, we obtain

$$
\begin{aligned}
& \frac{1}{2} \frac{d \overline{\delta\left\langle x_{i}(t)\right\rangle^{2}}}{d t}=\sigma_{\mathcal{E}}^{2} \frac{\bar{v}^{2} l_{c}^{2}}{7 D} \times \\
& \left(12 \sqrt{1+\frac{14 t}{3 \tau_{D}}}+\sqrt{1+\frac{7 t}{3 \tau_{D}}}+7 \sqrt{1+\frac{4 t}{\tau_{D}}}-13 \sqrt{1+\frac{13 t}{3 \tau_{D}}}-\sqrt{1+\frac{2 t}{\tau_{D}}}\right) .
\end{aligned}
$$

The effective dispersion coefficient $D_{11}^{\mathrm{eff}}(t)$ is obtained from (5.11) and (5.12) according to (4.13). For comparison, the unfiltered effective dispersion coefficient, i.e., for $\lambda \rightarrow \infty$, is given by (Clincy \& Kinzelbach 2001),

$$
D_{11}^{\mathrm{eff}}(t)=D+\sigma_{\mathcal{E}}^{2} \frac{\bar{v}^{2} l_{c}^{2}}{D}\left(2 \sqrt{1+\frac{2 t}{\tau_{D}}}-\sqrt{1+\frac{4 t}{\tau_{D}}}-1\right) .
$$

Notice that the ensemble and effective dispersion coefficients are independent of the distribution $c_{0}(\mathbf{x})$ of initial particle positions.

The behaviors for the filtered effective and ensemble dispersion coefficients in the early time regime $\left(6 D^{2} / l_{c}^{2} \bar{v}^{2} \sigma_{\mathcal{E}}^{2}\right)^{1 / 3} \tau_{D} \ll t \ll \tau_{D}$ are $D_{11}^{\text {ens }}(t) \sim D_{11}^{\text {eff }}(t) \sim\left(t / \tau_{D}\right)^{3}$. In the long time regime $t \gg \tau_{D}$, they show the characteristic scaling as $\left(t / \tau_{D}\right)^{1 / 2}$ (Matheron \& de Marsily 1980). The unfiltered effective dispersion coefficient (5.13) behaves at $t \ll \tau_{D}$ as $\left(t / \tau_{D}\right)^{2}$, which is characteristic of dispersion in a linear shear flow (Bolster et al. 2011). Thus, the unfiltered effective coefficient incorporates dispersion effects due to shear deformation, while its filtered counterparts are at the same time of the order of the local dispersion coefficient, i.e., unaffected by advective heterogeneity. Figure 4 illustrates the evolution of the filtered effective and ensemble dispersion coefficients as well as the unfiltered effective dispersion coefficient (5.13). The effective and ensemble dispersion coefficients are almost indistinguishable, i.e., the trajectory fluctuations are small compared to the ensemble dispersion coefficient. The evolution of the mixing scale-dependent dispersion coefficients reflects the sampling of the pertinent heterogeneity length scales. The dispersion coefficients are of the order of the local scale dispersion coefficient until 


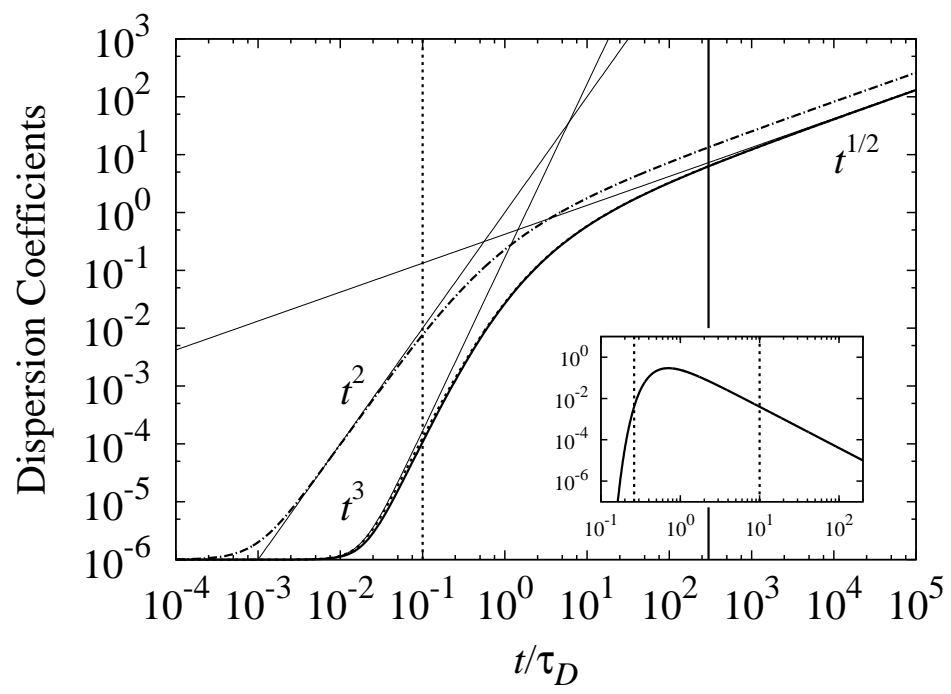

Figure 4. Mixing-scale dependent (solid lines) effective and (dashed lines) longitudinal ensemble dispersion coefficients for $P e=10^{3}$ in a $d=2$ dimensional stratified flow. The dash-dotted line represents the unfiltered effective dispersion coefficient. The dotted vertical line indicates the time scale $\tau_{c}=l_{m}^{2} / 6 D$, the solid vertical line the time scale $\hat{\tau}_{D}=\left(10 l_{c}\right)^{2} / 2 D$. The thin solid lines indicate the early time $t^{3}$ and late time $t^{1 / 2}$ behaviors of the filtered effective dispersion coefficient, respectively, as well as the $t^{2}$ behavior of the unfiltered effective dispersion coefficient. The dispersion coefficients are normalized by $\sigma_{\mathcal{E}}^{2} \bar{v}^{2} l_{c}^{2} / D$. The inset illustrates the scale distribution $p_{l}(l)$, the horizontal axis denotes $l / l_{c}$. The vertical lines indicate $l_{m}=0.26 l_{c}$ and $10 l_{c}$.

the mixing scale $\lambda\left(t=\tau_{c}\right)=l_{m}$ is of the order of the minimum heterogeneity scale defined in Section (3). This gives for the activation time scale $\tau_{c}=l_{m}^{2} /\left(2 D f_{\beta}\right)$ where here $\beta=1$. The dispersion coefficients increase for $t>\tau_{c}$ as the solute scans through the distribution of heterogeneity scales as indicated in the inset in Figure 4. For times $t>\left(10 l_{c}\right)^{2} /\left(2 D f_{\beta}\right)$ the mixing scale comprises 10 characteristic heterogeneity length $l_{c}$ and the filtered dispersion coefficients assume the characteristic correlation-induced $t^{1 / 2}$ scaling (Matheron \& de Marsily 1980; Bouchaud \& Georges 1990).

\subsection{Heterogeneous Porous Media Flow}

We consider now dispersion in the flow through heterogeneous media as described in Section 2. The transport problem does not possess a closed form solution for the dispersion coefficients, thus, we study the dispersion behavior in the frame of a second-order perturbation expansion in the fluctuations of the random velocity field $\tilde{\mathbf{v}}^{\prime}(\mathbf{k})$.

This implies the substitution of $\tilde{c}\left(\mathbf{k}, t \mid \mathbf{x}^{\prime}\right)$ in (4.6) and (4.8) by $\tilde{c}_{0}\left(\mathbf{k}, t \mid \mathbf{x}^{\prime}\right)=\langle\exp [i \mathbf{k}$. $\left.\left.\mathbf{x}_{0}(t)\right]\right\rangle$, where the 'unperturbed' particle trajectory is given by

$$
\mathbf{x}_{0}(t)=\mathbf{x}^{\prime}+\overline{\mathbf{v}} t+\int_{0}^{t} d t^{\prime} \boldsymbol{\xi}\left(t^{\prime}\right)
$$

Executing the noise average gives for the propagator $\tilde{c}_{0}\left(\mathbf{k}, t \mid \mathbf{x}^{\prime}\right)$,

$$
\tilde{c}_{0}\left(\mathbf{k}, t \mid \mathbf{x}^{\prime}\right)=\exp \left(-\mathbf{k} \cdot \mathbf{D} \mathbf{k} t-i \overline{\mathbf{v}} \cdot \mathbf{k} t+i \mathbf{k} \cdot \mathbf{x}^{\prime}\right) .
$$

Using this approximation in (4.8), we obtain for the ensemble dispersion coefficients (4.14) 
the perturbation expression

$$
D_{i i}^{\mathrm{ens}}(t)=D+\int_{0}^{t} d t^{\prime} \int_{k} \mathcal{A}(\mathbf{k}, t) \mathcal{A}\left(\mathbf{k}, t^{\prime}\right) \mathcal{C}_{i i}(\mathbf{k}) \tilde{c}_{0}\left(-\mathbf{k}, t-t^{\prime}\right),
$$

which is similar to the one derived by Rubin et al. (1999) for a constant ideal filter. For the trajectory fluctuations, we derive by using (5.15) in (4.6) and (4.15) the expression

$$
\frac{1}{2} \frac{d \overline{\delta\left\langle x_{i}(t)\right\rangle^{2}}}{d t}=\int_{0}^{t} d t^{\prime} \int_{k} \mathcal{A}(\mathbf{k}, t) \mathcal{A}\left(\mathbf{k}, t^{\prime}\right) \mathcal{C}_{i i}(\mathbf{k}) \tilde{c}_{0}(\mathbf{k}, t) \tilde{c}_{0}\left(-\mathbf{k}, t^{\prime}\right) .
$$

The mixing scale $\lambda(t)$ is set to $\lambda(t)=\sqrt{2 D t f_{\beta}}$ in the filter (4.3).

Notice, that the scalar may starts sampling the flow heterogeneity earliest when it has been transported advectively over a disorder correlation length. Thus, we focus our analysis on times larger than the advection time scale $\tau_{v}$. Furthermore, in most practical applications transport is advection-dominated, that is, the Péclet number is $P e \gg 1$. Under these conditions, we obtain for the longitudinal ensemble dispersion coefficient the compact expression

$$
D_{11}^{\mathrm{ens}}(t)=D+\sqrt{\frac{\pi}{2}} \sigma_{\mathcal{E}}^{2} \bar{v} l_{c}\left[1+\left(1+\frac{4 t f_{\beta}}{\tau_{D}}\right)^{-\frac{d-1}{2}}-2\left(1+\frac{2 t f_{\beta}}{\tau_{D}}\right)^{-\frac{d-1}{2}}\right] .
$$

The approximation method leading to this expression is outlined in Dentz et al. (2000). The first term in the square brackets quantifies the unfiltered ensemble dispersion coefficient. Notice that lowest order perturbation theory renders the transverse dispersion coefficients of the order of the inverse Péclet number. Thus we focus here on the longitudinal coefficients only. Note that at times $t \ll \tau_{D}$, the mixing-scale dependent ensemble dispersion coefficient is significantly reduced compared to its unfiltered counterpart, which evolves to its asymptotic value on the advection time scale $\tau_{v}$ (Dagan 1991).

The evolution of the trajectory fluctuations, which quantify the difference between them, see (4.15), are obtained as

$$
\begin{aligned}
\frac{1}{2} \frac{d \overline{\delta\left\langle x_{1}(t)\right\rangle^{2}}}{d t}= & \sqrt{\frac{\pi}{2}} \sigma_{\mathcal{E}}^{2} \bar{v} l_{c}\left\{\left(1+\frac{4 t}{\tau_{D}}\right)^{-\frac{d-1}{2}}+\right. \\
& {\left.\left[1+\frac{4 t\left(1+f_{\beta}\right)}{\tau_{D}}\right]^{-\frac{d-1}{2}}-2\left[1+\frac{\left(4+2 f_{\beta}\right) t}{\tau_{D}}\right]^{-\frac{d-1}{2}}\right\} }
\end{aligned}
$$

The expression on the first line quantifies the trajectory fluctuations with respect to the ensemble average flow velocity $\bar{v}$ (Dentz et al. 2000). As in the previous section, both the effective and ensemble dispersion coefficients are independent of the distribution of initial particle positions $c_{0}(\mathbf{x})$.

Figure 5 illustrates the evolution of the mixing-scale dependent effective and ensemble dispersion coefficients. The mixing-scale dependent effective and ensemble dispersion coefficients evolve much slower than their unfiltered effective counterpart. The unfiltered effective dispersion coefficient, picks up for times smaller than the advection time scale $\tau_{v}$. This behavior can be traced back to advective spreading of the scalar distribution as its size increases due to dispersion. The filtered dispersion coefficients, in contrast, remain essentially at the value of the local dispersion coefficient until the time $\tau_{c}$ which depends on the compression factor $f_{\beta}$ and the smallest heterogeneity scales. As above, it is set 


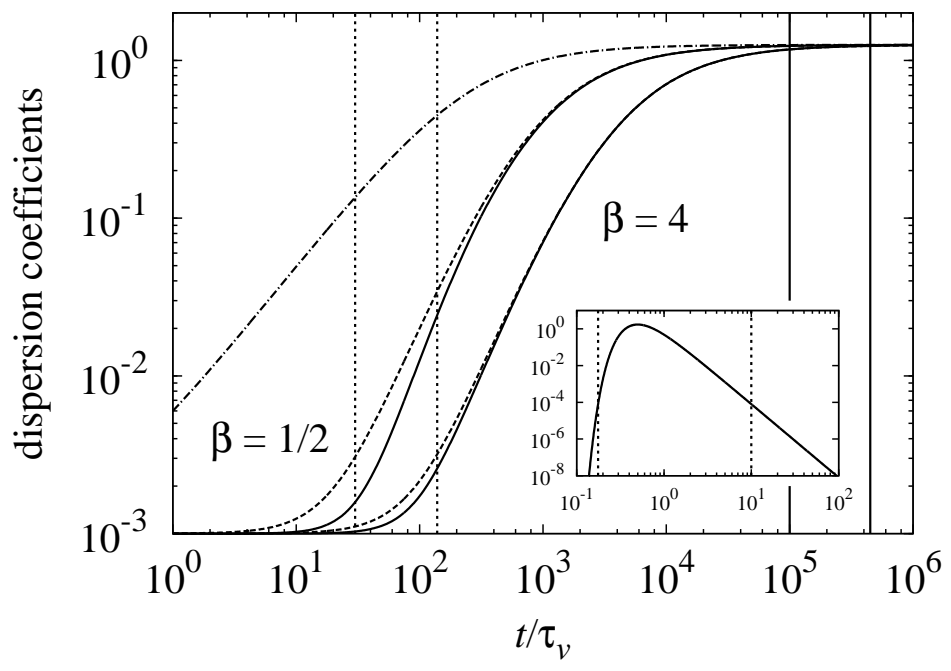

Figure 5. Mixing-scale dependent (solid lines) effective and (dashed lines) longitudinal ensemble dispersion coefficients for $P e=10^{3}$ and $d=3$ spatial dimensions. The dash-dotted line represents the unfiltered effective dispersion coefficient. The dotted vertical lines indicate the time scale $\tau_{c}=l_{m}^{2} / 2 D f_{\beta}$ for $\beta=1 / 2$ and $\beta=4$. The solid vertical lines denote the time scale $\hat{\tau}_{D}=\left(10 l_{c}\right)^{2} / 2 D f_{\beta}$. The dispersion coefficients are rescaled by $\sigma_{\mathcal{E}}^{2} \bar{v} l_{c}$. The inset illustrates the scale distribution $p_{l}(l)$, the horizontal axis denotes $l / l_{c}$. The vertical lines indicate $l_{m}=0.18 l_{c}$ and $10 l_{c}$.

by the heterogeneity scale $l_{m}$ through $\lambda\left(\tau_{c}\right)=l_{m}$, and equivalently $\tau_{c}=l_{m}^{2} /\left(2 D f_{\beta}\right)$. For $t<\tau_{c}$, i.e., for scales smaller than $l_{m}$, the mixing scale is smaller than the minimum heterogeneity scale. Thus the flow field is homogeneous, and therefore dispersion is equal to local dispersion. For $t>\tau_{c}$, heterogeneity is activated as a dispersion mechanism. As the mixing scale increases, it scans through the distribution of heterogeneity scales, illustrated in the inset in Figure 5 and incorporates fluctuations of increasing scale into the dispersion process. For times $t \gg \tau_{c}$, the dispersion coefficients evolve towards their asymptotic long-time value. For times $t \gg \tau_{D}$, that is, as the mixing scale $\lambda(t) \gg l_{c}$, the full spectrum of velocity values is available and the velocity fluctuations that cause dispersion are statistically equal in all realizations.

\section{Conclusions}

The impact of velocity fluctuations on scalar dispersion in heterogenous flow fields has been quantified in terms of macroscale dispersion coefficients. The concept of macrodispersion assumes that all velocity fluctuations scales are equally available for solute dispersion, while blockscale dispersion quantifies the impact of velocity fluctuations below a fixed coarse-graining scale on effective scalar transport.

We propose a dispersion approach for transport in spatially heterogeneous steady flow fields that integrates only velocity fluctuations below the mixing scale. The mixing scale aligns the physical length scales below which the scalar mixture can be assumed to be well mixed. It delineates the fluctuation length scales to which solute particles have statistically equal access. The mixing scale evolves through the competition of dispersive 
expansion and compression due the deformation of material fluid elements, which provide the support for scalar transport.

The behavior of the mixing-scale dependent dispersion coefficients reflects the assimilation of heterogeneity scales through the evolving mixing scale. It is determined on one hand by the competition between local scale dispersion and the compression of material fluid elements, which govern the mixing scale behavior, and on the other hand by the distribution of velocity scales, which are encoded in the velocity power spectrum. We study this behavior for dispersion in random shear flow and in heterogeneous porous media using a stochastic modeling approach. The filtered dispersion coefficients are of the order of the local scale dispersion coefficients as the mixing scale is smaller than a characteristic minimum fluctuation length scales. Then, as the scalar scans through the distribution of velocity scales, i.e., as more of the variability of the flow field becomes available for dispersive particle motion, the filtered dispersion coefficients increase towards their asymptotic behavior that is approximated at times, for which the mixing scale $\lambda(t)$ is much larger than the characteristic fluctuation scale $l_{c}$.

The interaction of scalar and heterogeneity length scales plays a fundamental role for the understanding of mixing and dispersion processes in heterogeneous media, and thus for the quantification of scalar fluctuations and fast mixing-limited reactions (De Simoni et al. 2005) in heterogeneous porous media. The proposed mixing-scale dependent dispersion concept may provide an operational tool to quantify these processes for transport in heterogeneous flows in the context of effective large scale transport models.

The authors thank three anonymous reviewers for their insightful comments. MD acknowledges the support of the European Research Council (ERC) through the project MHetScale (617511).

\section{REFERENCES}

de Barros, F.P.J., Dentz, M., Koch, J. \& NowaK, W. 2012 Flow topology and scalar mixing in spatially heterogeneous flow fields. Geophys. Res. Lett. 39 (8), L08404.

DE BARros, F.P.J. \& Rubin, Y. 2011 Modelling of block-scale macrodispersion as a random function. J. Fluid Mech. 676, 514-545.

BAtchelor, G.K. 1959 Small-scale variation of convected quantities like temperature in turbulent fluid part 1. general discussion and the case of small conductivity. J. Fluid Mech. 5 (01), 113-133.

Bear, J. 1972 Dynamics of Fluids in Porous Media. American Elsevier, New York.

BECKIE, R. 1996 Measurement scale, network sampling scale, and groundwater model parameters. Water Resour. Res. 32 (1), 65-76.

Beckie, R., Aldama, A. \& Wood, E.F. 1996 Modeling the large-scale dynamics of saturated groundwater flow using spatial-filtering theory: 1 . theoretical development. Water Resour. Res. 32 (5), 1269-1280.

Bolster, D., Dentz, M. \& Le Borgne, T. 2011 Hyper mixing in shear flow. Water Resour. Res. 47, W09602.

Bouchaud, J-P. \& Georges, A. 1990 Anomalous diffusion in disordered media: statistical mechanisms, models and physical applications. Phys. Rep. 195 (4), 127-293.

Brenner, H. 1980 Dispersion resulting from flow through spatially periodic porous media. Philos. Trans. Roy. Soc. London Ser. A 297 (1430), 81-133.

Clincy, M. \& Kinzelbach, H. 2001 Stratified disordered media: exact solutions for transport parameters and their self-averaging properties. J. Phys. A: Math. Gen. 34 (36), 7141.

Dagan, G. 1987 Theory of solute transport by groundwater. Annu. Rev. Fluid Mech. 19 (1), $183-213$.

DAGAN, G. 1991 Dispersion of a passive solute in non-ergodic transport by steady velocity fields in heterogeneous formations. J. Fluid Mech. 233, 197-210. 
De Simoni, M., Carrera, J., Sánchez-Vila, X. \& Guadagnini, A. 2005 A procedure for the solution of multicomponent reactive transport problems. Water Resour. Res. 41, 2005 WR004056.

Dentz, M., Kinzelbach, H., Attinger, S. \& Kinzelbach, W. 2000 Temporal behavior of a solute cloud in a heterogeneous porous medium: 1. point-like injection. Water Resour. Res. 36 (12), 3591-3604.

Dentz, M., Le Borgne, T., Englert, A. \& Bijeluic, B. 2011 Mixing, spreading and reaction in heterogeneous media: A brief review. J. Contam. Hydrol. 120, 1-17.

Duplat, J., InNocenti, C. \& Villermaux, E. 2010 A nonsequential turbulent mixing process. Phys. Fluids 22, 035104.

Ferziger, J.H. \& Perić, M. 2002 Computational methods for fluid dynamics, , vol. 3. Springer Berlin.

FIORI, A. 1998 On the influence of pore-scale dispersion in nonergodic transport in heterogeneous formations. Transport Porous Med. 30 (1), 57-73.

Gelhar, L.W. \& Axness, C.L 1983 Three-dimensional stochastic analysis of macrodispersion in aquifers. Water Resour. Res. 19 (1), 161-180.

Hornung, Ulrich 1997 Homogenization and Porous Media. Springer-Verlag New York, Inc.

Kitanidis, P. 1988 Prediction by the method of moments of transport in a heterogeneous formation. J. Hydrol. 102 (1), 453-473.

Kubo, R., Toda, M. \& Hashitsume, N. 1991 Statistical physics ii: Nonequilibrium statistical physics. Springer Series in Solid-State Sciences 31.

Le Borgne, T., Dentz, M., Davy, P., Bolster, D., Carrera, J., De Dreuzy, J-R. \& Bour, O. 2011 Persistence of incomplete mixing: A key to anomalous transport. Phys. Rev. E 84 (1), 015301.

Le Borgne, T., Dentz, M. \& Villermaux, E. 2013 Stretching, coalescence, and mixing in porous media. Phys. Rev. Lett. 110 (20), 204501.

Le Borgne, T., Dentz, M. \& Villermaux, E. 2015 The lamellar description of mixing in porous media. J. Fluid. Mech. .

Matheron, M. \& De Marsily, G. 1980 Is transport in porous media always diffusive? Water Resour. Res. 16, 901-917.

Mehrabi, A.R. \& SAhimi, M. 1997 Coarsening of heterogeneous media: application of wavelets. Phys. Rev. Lett. 79 (22), 4385.

Meunier, P. \& Villermaux, E. 2010 The diffusive strip method for scalar mixing in two dimensions. J. Fluid Mech. 662, 134-172.

RANZ, W. E. 1979 Application of a stretch model to mixing, diffusion and reaction in laminar and turbulent flows. AIChE Journal 25 (1), 41-47.

Risken, H. 1996 The Fokker-Planck Equation. Springer Heidelberg New York.

Rubin, Y 2003 Applied stochastic hydrogeology. Oxford University Press, New York.

Rubin, Y., Sun, A., Maxwell, R. \& Bellin, A. 1999 The concept of block-effective macrodispersivity and a unified approach for grid-scale-and plume-scale-dependent transport. $J$. Fluid Mech. 395, 161-180.

TAYLOR, G. 1953 Dispersion of soluble matter in solvent flowing slowly through a tube. Philos. Trans. Roy. Soc. London Ser. A 219 (1137), 186-203.

Tél, T., De Moura, A., Grebogi, C. \& KÁrolyi, G. 2005 Chemical and biological activity in open flows: a dynamical system approach. Phys. Rep. 413 (2), 91-196.

Tennekes, H. \& Lumley, J. L. 1972 A First Course in Turbulence. MIT Press, Cambridge,Mass.

Villermaux, E. 2012 Mixing by porous media. C. R. Mecanique 340 (11), 933-943.

Villermaux, E. \& Duplat, J. 2003 Mixing as an aggregation process. Phys. Rev. Lett. 91 (18), 184501

Villermaux, E. \& Duplat, J. 2006 Coarse grained scale of turbulent mixtures. Phys. Rev. Lett. 97 (14), 144506.

Weiss, J.B. \& Provenzale, A. 2008 Transport and mixing in geophysical flows, , vol. 744. Springer.

Whitaker, S. 1999 The Method of Volume Averaging. Kluwer Academic Publishers.

Wood, B.D., Cherblanc, F., Quintard, M. \& Whitaker, S. 2003 Volume averaging for 
determining the effective dispersion tensor: Closure using periodic unit cells and comparison with ensemble averaging. Water Resour. Res. 39 (8).

Zavala-Sanchez, V., Dentz, M. \& Sanchez-Vila, X. 2009 Characterization of mixing and spreading in a bounded stratified medium. Adv. Water Resour. 32, 635-648. 